\title{
Caracterización y principales patologías de la arenisca "Piedra Dorada" empleada en los edificios históricos de las ciudades de Úbeda y Baeza (Jaén, Sur de España)
}

\author{
J. Gisbertt ${ }^{(1,6)}$, R. Navarro ${ }^{(2,6)}$, J. Sánchez-Valverde ${ }^{(3)}$, J.M. Baltuille ${ }^{(4,6)}$ y E. Sebastián-Pardo ${ }^{(5,6)}$
}

(1) Dpto. de Ciencias Geológicas. Universidad de Zaragoza. C/Pedro Cerbuna s/n, 50009 Zaragoza, España. gisbert@posta.unizar.es.

(2) Instituto Geológico y Minero de España (IGME). Urb. Alcázar del Genil, 4, 18006 Granada, España. rafanavarro74@gmail.com

(3) Instituto Geológico y Minero de España (IGME). Litoteca de Peñarroya. Ctra. Estación, s/n. Pol. La Papelera. 14200 Peñarroya - Córdoba. josefina.sanchez@igme.es

(4) Dpto. de Infraestructura Geocientífica y Servicios. Instituto Geológico y Minero de España (IGME). Ríos Rosas, 23. 28003 Madrid, España. jmbaltuille@igme.es.

(5) Dpto. de Mineralogía y Petrología. Universidad de Granada. Avda. Fuente Nueva s/n, 18002 Granada, España. rolando@ugr.es.

(6) Red Construrock (Red de la Piedra Natural y su relación con el Patrimonio Histórico-Monumental y la obra nueva, en el ámbito del Estado español). Ríos Rosas, 23, 28003 Madrid, España.

\section{RESUMEN}

La arenisca conocida como "Piedra Dorada" es uno de los materiales más empleados en la construcción de gran parte de los edificios históricos de la provincia de Jaén. Un ejemplo de ello se encuentra en las ciudades de Úbeda y Baeza, declaradas Patrimonio Cultural de la Humanidad por la Unesco. Se ha realizado una caracterización detallada de la roca, en sus principales variedades: "Viva", "Franca" y "Jabaluna". Ha sido analizado tanto material de las canteras originales como muestras de monumentos. En el presente trabajo se determinan las principales características mineralógicas, texturales y geoquímicas así como las propiedades físicas de las variedades de "Piedra Dorada". Además se profundiza en el estudio de las principales patologías encontradas en los edificios históricos de las ciudades anteriormente citadas. A partir de los diferentes parámetros determinados se ha establecido un orden de calidad de las variedades de "Piedra Dorada". También se han caracterizado los mecanismos de deterioro entre los que se encuentran: colonización biológica (líquenes, algas, musgos y plantas superiores), ascenso capilar, depósitos superficiales (sales y costras negras) y deterioros debidos a factores antrópicos como fugas en la red de alcantarillado, ausencia de cubiertas y bajantes, etc. Este estudio ha permitido conocer en detalle una de las piedras de construcción más importantes de Andalucía con el fin de garantizar su perdurabilidad a través de los años.

Palabras clave: arenisca, durabilidad, patrimonio arquitectónico, Piedra Dorada, roca ornamental.

\section{Characterization and main pathologies of the sandstone "Piedra Dorada" used in historic buildings in the cities of Úbeda and Baeza (Jaén, southern Spain)}

\begin{abstract}
The sandstone known as "Piedra Dorada", or golden stone, is one of the most commonly used materials in the construction of many of the main historic buildings in the province of Jaén. Many examples can be found in the cities of Úbeda and Baeza, declared World Heritage Cities by UNESCO. A thorough characterization of their main varieties: "Viva", "Franca" and "Jabaluna" has been done. Original quarry materials as well as samples of monuments have been analyzed. In this study we present a characterization of the mineralogical, textural and geochemistry features as well as the main physical properties of the varieties of "Piedra Dorada." Furthermore, this research investigates in depth the main pathologies found in historic buildings in the cities
\end{abstract}


mentioned above. We have established an order of quality of the varieties mentioned from the different parameters determined. We have also characterized the deterioration mechanisms amongst which are: biological colonization (lichens, algae, moss and higher plants), an increase in capillary action, surface deposits (salts and black crust) and anthropic factors such as leaks into the sewer network, absence of covers and downspouts, etc. This study has allowed us to know in detail one of the most important construction stones of Andalusia in order to ensure its durability for future years.

Key words: architectonic heritage, durability, natural stone, Piedra Dorada, sandstone.

\section{ABRIDGED ENGLISH VERSION}

\section{Introduction}

Sandstone is a sedimentary stone widely used as a building material (Cultrone et al., 2012) which, like all stone materials can present problems of decay that vary depending on the composition, texture and environmental conditions (Charola, 2004). Some of the main problems of alteration that these stones present are the formation of surface deposits (salts and black crust) as well as biological colonization, among others.

Important examples of the use of sandstone are located in the province of Jaén, especially in the towns of Úbeda and Baeza. These cities were declared World Heritage Cities by UNESCO on July 3, 2003. Most of the monuments have been built with a bioclastic calcareous sandstone traditionally known as "Piedra Dorada" or golden stone that was extracted in the area.

There are three different varieties of "Piedra Dorada" (Alcalde et al., 1998): "Viva", "Franca" and "Jabaluna" The first is more cemented and resistant and used for structural purposes; the second, more porous and less cemented, and has traditionally been used in decoration. The third variety called "Jabaluna", whose main feature is a blue-yellow banded colour (Alcalde et al., op. cit.), is more similar to the "Viva" variety, although it is less used due to its reduced availability.

The aim of this study is to characterize the main mineralogical and physical properties as well as the main pathologies the "Piedra Dorada" presents, as it is one of the most important natural stones of the architectural heritage of Andalusia.

\section{Materials and Methods}

From a geological point of view, the "Piedra Dorada" is a bioclastic calcareous sandstone, with a fine to medium grain size, which belongs to the deposits of the Guadalquivir Basin, Upper Tortonian or Messinian (Postorogenic Neogene Basin of the Betic cordillera) (Roldan, 2011).

Currently there are no traces of the historic quarries that supplied the materials for the construction of the monuments of Úbeda and Baeza, because these quarries have been anthropised for olive cultivation. Therefore, samples of the "Viva" (Fig. 1 a) and "Franca" (Fig. 1 b) varieties from Porcuna were taken from a sandstone quarry in Porcuna (Fig. 1 c) called Aguilera de Dios, S.L. (Long: $4{ }^{\circ} 10$ ' 46 "N; Lat: $37^{\circ} 52^{\prime} 04^{\prime \prime}$ W), whilst samples of the "Jabaluna" varieties from the area of Baeza and "Viva" and "Franca" from the area of Sabiote were supplied by the company "Piedras Sujas".These quarries use the same materials that were utilized in the construction of the historical buildings. Furthermore, samples from some buildings, such as the Arch of Villarar or the Gate of Jaén (Baeza) as well as alterations in monuments such as the Town Hall, the Church of San Salvador, the Church of San Pablo and the Palace of the Counts Guadiana in Úbeda and the Convent of San Francisco, the Town Hall and the Cathedral in Baeza were taken.

The petrographic characterization of quarry material (JA1 to JA5) has been done with a petrographic polarizing microscope (Leica DM2500P) with an incorporated digital camera (LEICA DFC450). Chemical analysis was made using an atomic absorption spectrometer, Perkin-Elmer model 2380. As far as the hydric properties are concerned, we determined the porosity, bulk density (UNE-EN 1936), absorption of water at atmospheric pressure (UNE-EN 13755) and desorption of liquid water at atmospheric pressure and at room temperature. We have also tested resistances to salt crystallization (RILEM, 1980 V.1b). Samples of alterations in monuments have been studied by $X$-ray diffraction and scanning electron microscopy (SEM).

\section{Results and discussion}

Table 1 shows the petrographic properties and chemical analysis of quarry samples of "Piedra Dorada". Optical microscopy shows that the rock is composed of more than $50 \%$ of thin-shelled lamellibranch frag- 
ments, rounded and equigranular quartz grains and carbonated clasts and a highly carbonated cementation (Fig. 2). It has a fine to medium grain size which is fairly homogeneous (Gisbert et al., 2007). A small percentage $(<11 \%)$ of other minerals appear, such as Fe oxides, glauconite, feldspar or pyrolusite. It is noteworthy that, depending on the quarry of origin, samples of the variety "Viva" and "Jabaluna" from Baeza and Sabiote (JA.1 and JA.2) have a higher percentage of iron oxides, which is reflected in the colour of the sample, with darker tonalities, mainly brown tones. The Porcuna samples (JA.4) also have a higher proportion of dolomite fragments.

Regarding porosity, the "Jabaluna" and "Viva" varieties are less porous and mainly consist of micropores. They have a high percentage of calcite cement and a lower relative proportion of insoluble residue. The variety "Franca" has a high porosity and a dual porosity system, which is its greatest weakness, as the macropores make the penetration of water and salts into the micropores easier, where these components operate (Gisbert et al., op. cit.).

Regarding the hydric behaviour of the quarry samples, as shown in Table 2, the "Jabaluna" varieties (from Baeza) and "Viva" (from Sabiote and Porcuna) have a reduced porosity accessible to water, below $7 \%$ compared to values higher than $22 \%$ reached by "Franca" variety (from Sabiote and Porcuna). As to the water penetration kinetics (Fig. 3 a), the "Jabaluna" and "Viva" varieties have low water penetration rate and low absolute water absorption percentage. In contrast, the "Franca" variety stands out for the high absolute values reached and for the high speed of penetration of water inside. The desorption test provides us with information regarding these aspects (Fig. 3 b). In the "Jabaluna" and "Viva" varieties, loss of water retained in the pore systems is very slow, the opposite to that observed in the "Franca" variety. This information confirms the remark that the porous system is very different both in quantity and tortuosity, so the percentage and degree of interconnection is much higher in the "Franca" variety.

About decay behaviour, salt crystallization test results indicate a direct relationship between the percentage of open porosity and the decay suffered by the samples (Table 3). Therefore, the "Jabaluna" and "Viva" varieties (Fig. $4 a, b, c)$ have less pronounced damage than the "Franca" variety (Fig. $4 d$, e).

As for the main pathologies in the buildings studied (Fig. 5), biological colonization (lichens, algae, mosses and higher plants), humidity and surface deposits (salts and black crusts) should be highlighted.

The black crust sample from the Church of San Salvador (Úbeda) (U.1) has a high degree of biological colonization in both density and depth (Fig. 6 a). The U.2 sample from the same church, has gypsum crystals, spherical particles of pollution and biological remains (Fig. 6 b, c), although poorly developed. The B.2 sample has a similar composition to the latter, though more developed, which has led to the disintegration of the stone (Fig. 6 d).

In relation to salt efflorescence, analysis by X-ray diffraction shows the main phases found are mainly magnesium sulphate (hexahedrite and epsomite), niter (sodium nitrate, $\mathrm{KNO}_{3}$ ), quartz and calcite which appear in almost every sample (Table 4). Other minerals present are potassium nitrate ( $\mathrm{NaNO}_{3}$ ) and $\mathrm{gypsum}$ $\left(\mathrm{CaSO}_{4}\right)$, found in one sample each. The possible origin of these salts is the capillary rise through the substrate that provides the nitrates and sulphates and washed areas rich in sulphates and nitrates, such as areas filled with Portland cement. Magnesium abundance in the stone produces magnesium salts which have a high hygroscopicity. This results in the presence of wet spots which can be removed only by withdrawal of these salts from inside the wall.

\section{Introducción}

La arenisca es una roca sedimentaria ampliamente utilizada como roca de construcción (Cultrone et al., 2012) que al igual que todos los materiales pétreos puede presentar problemas de alteración. Varían en función de la composición, de la textura y de las condiciones ambientales (Charola, 2004). Estas rocas, debido a las buenas características que presentan para su empleo como roca de construcción han sido ampliamente utilizadas en la herencia arquitectónica de Andalucía (Urosevic et al., 2011).

Son materiales que han sido muy estudiados, tanto desde el punto de vista de sus características mineralógicas y físicas como desde el punto de vista de las alteraciones y patologías que presentan, por numerosos autores (Alcalde et al., 1998; Campos y de la Torre, 2006 y 2007; Cultrone et al., op. cit.; Luque et al., 2008; Molina et al., 2011 y 2013; Ruiz Agudo et al., 2011; Rodríguez-Navarro, 1994; Sebastián-Pardo et al., 1995 y 2008; Urosevic et al., op.cit y 2012).

Algunos de los principales problemas de alteración que presentan estas rocas son la formación depósitos superficiales (sales y costras negras) así como la colonización biológica. El deterioro por sales es uno de los mayores mecanismos de degradación que afectan a los edificios históricos (RodríguezNavarro y Doehne, 1999), especialmente cuando las sales son muy solubles en agua y son transportadas al interior del sistema poroso de la roca (Ruiz-Agudo 
et al., op.cit. Por otro lado la formación de costras negras es un proceso de degradación especialmente importante en entornos urbanos donde la mezcla de partículas procedentes de la combustión de combustibles fósiles junto con otra variedad de factores como el polvo, la fauna microbiana, etc. (Watt et al., 2009) favorecen la formación de costras de yeso (Urosevic et al., 2012). También, la colonización biológica juega un papel fundamental en el deterioro (SaizJiménez y Ariño, 1995). Los líquenes ejercen un daño químico y físico sobre el sustrato además de un efecto estético negativo. La descomposición mecánica se produce porque los líquenes incrementan su masa conforme crecen, por otro lado, cambian de volumen con la humedad ambiental. Los fenómenos de contracción (período seco)-expansión (período húmedo) provocan el deterioro físico del sustrato. Químicamente, los líquenes producen ácidos orgánicos que son responsables de la degradación de la roca. Estos organismos favorecen la condensación de agua y su retención sobre la superficie pétrea (Nimis et al., 1992).

Un importante ejemplo del uso de la arenisca se encuentra en las ciudades de Úbeda y Baeza (Jaén). Estas ciudades fueron declaradas Patrimonio Cultural de la Humanidad por la Unesco el 3 de julio de 2003. La gran mayoría de sus monumentos han sido construidos con una arenisca calcárea que tradicionalmente se conoce como "Piedra Dorada", denominada así por su color amarillento (Sebastián-Pardo et al., 1995). Estas ciudades viven un gran periodo de esplendor económico durante el siglo XVI, especialmente debido a la instauración de una gran número de órdenes religiosas a partir de finales del s. XV y durante el s. XVI (García-Torralbo, 1998), ligado al cual se construyen los edificios renacentistas más destacados como son el Palacio de Vázquez de Molina o Palacio de las Cadenas (s.XVI), la Iglesia de San Salvador (s.XVI), o la Real Colegiata de Santa María la Mayor de los Reales Alcázares (s.XVI) en Úbeda o la Casa de la Justicia o del Corregidor (s.XVI), la Santa Catedral de Natividad de Nuestra Señora (s.XVI) o el Palacio de Jabalquinto (s. XV) en Baeza, o recientemente, la Casa de la Piedra (1931-1960) en Porcuna.

Dentro de la "Piedra Dorada" se diferencian tres variedades (Alcalde et al., idem.): "Piedra Viva", "Piedra Franca" y "Piedra Jabaluna". La primera de ellas, más cementada y resistente, se empleó principalmente en la elaboración de sillares y elementos estructurales; la segunda, algo más porosa y menos cementada, se ha empleado tradicionalmente en la elaboración de elementos decorativos (estatuas, pináculos, etc.), ya que es más fácil de labrar; por último se diferencia otra variedad denominada
"Jabaluna", cuya característica principal es un bandeado de diferentes colores, unos azules de aspecto pizarroso y otros amarillos (Alcalde et al., idem) y de características más parecidas a la variedad "Viva", que hace que se haya empleado como elementos de sustentación como columnas, capiteles, plintos, etc. aunque en menor proporción que las anteriores, ya que su disponibilidad es menor.

El objetivo de este trabajo es exponer las principales características de las tres variedades de "Piedra Dorada" que pueden influir en su comportamiento como roca de construcción y detallar las principales patologías que presentan los monumentos de las ciudades de Úbeda y Baeza (Jaén).

\section{Materiales y métodos}

\section{Localización geográfica y contexto geológico}

Las ciudades de Úbeda y Baeza se encuentran en la provincia de Jaén (Sur de España) y forman parte de la denominada comarca de la Loma.

Desde el punto de vista geológico, la "Piedra Dorada" es una arenisca calcárea bioclástica de grano fino a medio, que pertenece a los depósitos de una edad comprendida entre el Tortoniense Superior y el Messiniense de la Cuenca del Guadalquivir (Cuencas Neógenas Postorogénicas de las Cordilleras Béticas) (Roldán, 2011). A escala macroscópica es muy difícil distinguir las variedades "Jabaluna", "Viva" y "Franca". El color varía de marrón amarillento anaranjado (Viva-Franca) a gris verdoso (Jabaluna). Se presenta en estratos muy continuos que oscilan entre $10-20 \mathrm{~cm}$ de espesor con estratos margosos centimétricos intercalados. Sedimentológicamente son sistemas turbidíticos de la zona axial de la cuenca (foredeep) (Roldán, 1995). En la zona de Porcuna, los depósitos son de edad Messiniense, y la sucesión está formada por un intervalo inicial de unos $20 \mathrm{~m}$ de espesor compuesto por un horizonte arenoso, masivo, parcialmente cementado, con abundante contenido en bioclastos seguido de un tramo de unos $14 \mathrm{~m}$ de una alternancia de areniscas bioclásticas más o menos cementadas entre los que se intercalan niveles centimétricos de margas gris-azuladas; le sigue un tramo de unos 6 metros de alternancia rítmica de areniscas y margas gris-azuladas que da paso a unas margas gris-azuladas y blancas. En la zona de Sabiote, la secuencia es similar, con algunas variaciones de espesor, pero la edad es Tortoniense Superior (Roldán, idem). 


\section{Muestreo}

Actualmente no quedan vestigios de las canteras históricas que suministraron los materiales de construcción de los monumentos de Úbeda y Baeza, ya que ha reutilizado para el cultivo del olivar. No obstante, se han tomado muestras de canteras actuales localizadas en Sabiote y Porcuna, que explotan los mismos materiales que se emplearon. Además, para establecer una comparativa y poder valorar el estado de alteración que presentan los monumentos, se han tomado muestras de algunos edificios como el Arco de Villalar o la Puerta de Jaén (Baeza) así como de las alteraciones que presentan monumentos como el Ayuntamiento, la Iglesia de San Salvador, la Iglesia de San Pablo o el Palacio de los Condes de Guadiana en Úbeda y el Convento de San Francisco, el Ayuntamiento o la Catedral en Baeza.

Las muestras de cantera analizadas son la variedad "Jabaluna" (JA1), procedente de Baeza; variedad "Viva" (JA2), procedente de Sabiote; variedad "Franca" (JA3), procedente de Sabiote; variedad

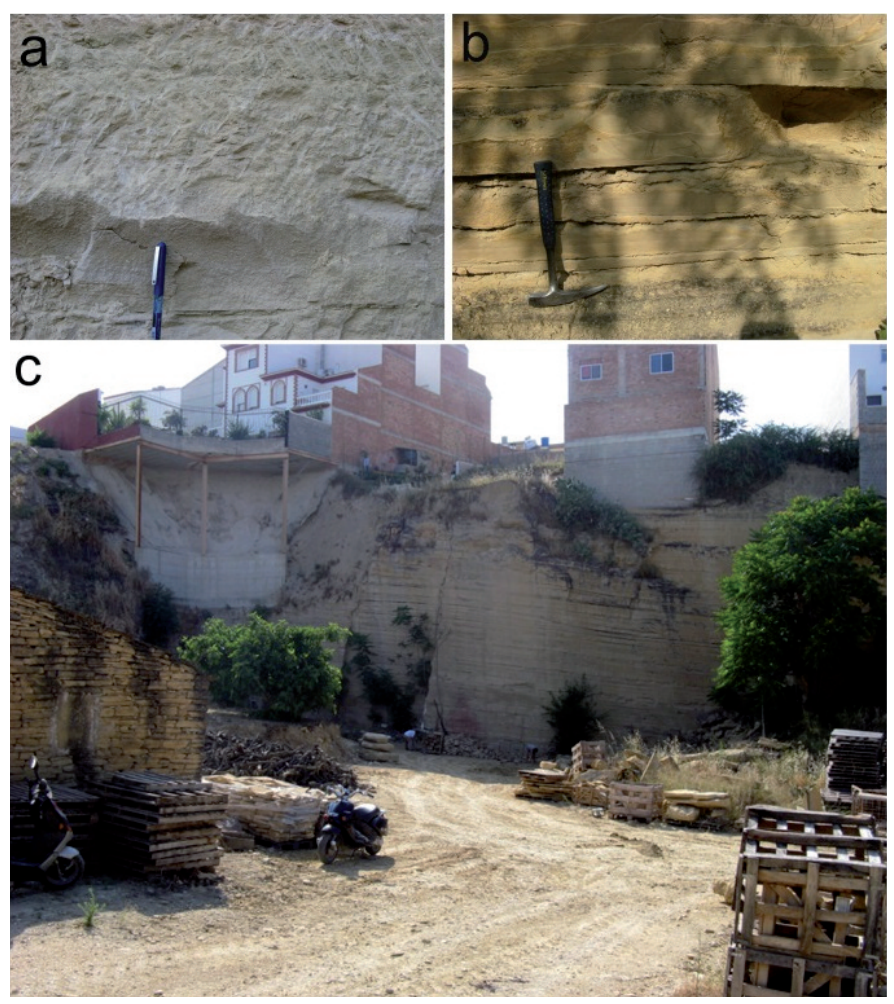

Figura 1. a) Detalle de la variedad "Viva"; b) detalle de la variedad "Franca"; c) Vista del estado actual de la cantera de Porcuna de la que se tomaron las muestras.

Figure 1. a) Detail of the variety "Viva"; b) detail of the variety "Franca"; c) view of the current state of the quarry in Porcuna from which the samples were collected.
"Viva" (JA4), procedente de Porcuna y variedad "Franca" (JA5), procedente de Porcuna. Las muestras de las variedades "Viva" (Fig. 1 a) y "Franca" (Fig. 1 b) procedentes de Porcuna se tomaron de la cantera "Areniscas de Porcuna" (Fig. 1 c) (Long: 4 $10^{\prime} 46^{\prime \prime} \mathrm{N}$; Lat: $37^{\circ} 52^{\prime} 04^{\prime \prime}$ ), de Aguilera de Dios, S.L. mientras que las muestras de las variedades "Jabaluna" procedente de la zona de Baeza y "Viva" y "Franca" procedentes de la zona de Sabiote fueron suministradas por la empresa de cantería "Piedras Sujas" de Sabiote, que no nos facilitó su procedencia exacta.

\section{Métodos analíticos}

Para evaluar las propiedades petrofísicas de esta variedad de roca así como para la caracterización de las alteraciones que presenta, se emplearon las siguientes técnicas:

1- Para la caracterización petrográfica del material de cantera (JA1 a JA5) se ha empleado un microscopio petrográfico de luz polarizada (MOP) Leica DM2500P con cámara digital incorporada (LEICA DFC450) y el correspondiente software de tratamiento de imágenes. La determinación de los porcentajes de los distintos componentes se realizó mediante contaje de puntos. Este método consiste en desplegar una malla de puntos con un espaciado constante sobre la superficie de la lámina, determinando en cada uno de ellos su composición. Para ciertos parámetros como la selección y la esfericidad y redondez de los fragmentos de árido, se han empleado una serie de valores procedentes de la comparación con gráficas de estimación visual (Beard y Weyl, 1977). El color se ha determinado mediante el sistema Munsell (Geological Society of America, 1991).

2- De las variedades de roca de cantera (JA1 a JA5) se han realizado análisis químicos de roca total. Se ha obtenido el residuo insoluble de la muestra frente a ataque ácido, el porcentaje de $\mathrm{CaO}, \mathrm{MgO}$ y $\mathrm{Fe}_{2} \mathrm{O}_{3}$ mediante análisis químico y el porcentaje de $\mathrm{H}_{2} \mathrm{O}, \mathrm{CO}_{2}$ y materia orgánica mediante pérdidas al fuego. Para ello en primer lugar se procedió a la molienda de las rocas en un molino de aros con mortero de acero hasta un tamaño de unas $100 \mu \mathrm{m}$. Las muestras se trataron mediante una extracción clorhídrica efectuada a partir de alícuotas de unos $250 \mathrm{mg}$ de muestra a las que se añadió $100 \mathrm{ml}$ de $\mathrm{HCl}$ $0,8 \mathrm{~N}$, en agitación continua durante 2 horas a $50{ }^{\circ} \mathrm{C}$. La solución resultante de este ataque se filtró con papel cuantitativo de bajo contenido 
en cenizas, se ajustó a volumen y se almacenó para su análisis. El Residuo Insoluble retenido en el papel de filtro se sometió a una temperatura de $900{ }^{\circ} \mathrm{C}$ para calcinar el papel y poder cuantificar el residuo resultante. Las determinaciones analíticas de los cationes se realizaron mediante espectrómetro de absorción atómica modelo Perkin-Elmer 2380. Por último, se calcularon las pérdidas de masa resultantes de someter a entre 10 y $15 \mathrm{~g}$ de muestra molida a temperaturas de 200,500 y $900{ }^{\circ} \mathrm{C}$. De esta manera se calculó el porcentaje de agua total, de materia orgánica y de dióxido de carbono respectivamente.

3- Se han determinado las principales propiedades hídricas de los materiales de cantera (muestras JA1 a JA5): porosidad abierta, la densidad aparente (UNE-EN 1936), la absorción de agua a presión atmosférica (UNE-EN 13755) y la desorción de agua líquida a presión atmosférica y temperatura ambiente. Para ello se han empleado cuatro probetas cúbicas de $5 \mathrm{~cm}$ de arista de cada una de las muestras de cantera (JA1 a JA5), siguiendo lo establecido en las normas UNE de referencia.

4- Con respecto a los ensayos de envejecimiento acelerado se han realizado ensayos de resistencia a la cristalización de sales siguiendo lo establecido en la norma RILEM, 1980, V.1b. Para ello se han realizado 3 probetas cúbicas de cada una de las muestras de cantera (JA1 a JA5). Se han realizado una serie de 15 ciclos de 24 horas. Se han sumergido las probetas en una disolución de sulfato sódico decahidratado $\left(\mathrm{NaSO}_{4} * 10 \mathrm{H}_{2} \mathrm{O}\right)$ diluido al $14 \%$, durante 4 horas. Seguidamente se introducen en una estufa a $105{ }^{\circ} \mathrm{C}$ durante 16 horas y, por último, se dejan enfriar las probetas en un desecador a temperatura ambiente durante 4 horas, para evitar los deterioros debidos al choque térmico.

5- En lo referente a las principales alteraciones, la caracterización mineralógica de las sales de las eflorescencias de monumentos se ha realizado por difracción de rayos $\mathrm{X}$ empleando para ello un difractómetro Rigaku, modelo D/max 2500 provisto de un ánodo de cobre rotante ( $\mathrm{K}$ del $\mathrm{Cu},=1,5405$ ) y monocromador de grafito. Se emplearon $40 \mathrm{kV}$ de voltaje y $80 \mathrm{~mA}$ de intensidad, con un ángulo de barrido $(2 \theta)$ de $10^{\circ}$ a $80^{\circ}$, con un paso de $0,03 \%$ seg. Para la determinación de fases se ha utilizado la base de datos "JCPDS-International Centre for Diffraction Data-2000. Se han analizado muestras procedentes del lado sur del Palacio de los Condes de
Guadiana (U.5), lado noreste de la Iglesia de San Pablo (U.7) y lado norte del Ayuntamiento (U.23), en Úbeda, y ya en Baeza, el interior del Convento de San Francisco (B.37), el lado norte de la Catedral (B.6) y el lado sureste del Ayuntamiento (B.1).

6- El estudio de las costras negras de los monumentos se ha realizado por microscopía electrónica de barrido. Se ha empleado el equipo JEOL JSM 6400 con detector de energía de rayos $\mathrm{X}$ dispersados eXL-10 para la realización de análisis químicos puntuales. Se han analizado un total de tres muestras procedentes de dos monumentos: U.1: muestra de costra negra desarrollada sobre arenisca en el lado norte de la iglesia de San Salvador (Úbeda); U.2: muestra de costra negra desarrollada sobre mortero en la portada norte de la iglesia de San Salvador (Úbeda) y B.2: muestra de costra negra desarrollada sobre arenisca en la esquina noroeste del Arco de Villalar (Baeza).

Estos ensayos se han llevado a cabo en el laboratorio del Área de Petrología del Departamento de Ciencias de la Tierra de la Universidad de Zaragoza.

\section{Resultados y discusión}

\section{Petrología}

Al microscopio óptico se observa que la roca está compuesta por más del $50 \%$ de fragmentos de lamelibranquios de concha fina, granos de cuarzo equigranulares y redondeados y clastos carbonatados y una cementación carbonatada elevada (Fig. 2). Presentan un tamaño de grano fino a medio (desde 0,125-250 a $0,5 \mathrm{~mm}$ ), bastante homogéneo (Gisbert et al., op. cit). En mucha menor medida $(<11 \%)$ aparecen otros minerales como óxidos de Fe, glauconita, feldespatos o pirolusita. Según Sebastián-Pardo et al. (1995), la presencia de glauconita es la causante de que esta arenisca muestre el color dorado al que debe su nombre. En la tabla 1 se muestran los resultados de la caracterización mineralógica y los análisis químicos de las muestras de cantera.

Se observa que, en función de la cantera de procedencia, las muestras de la variedad "Jabaluna" y "Viva" de Baeza y Sabiote (JA.1 y JA.2) presentan mayor porcentaje de hierro, bien como hierro ferroso en la red de la calcita, como hierro ferroso y férrico en la glauconita o bien como $\mathrm{Fe}^{3+}$ en forma de óxidos e hidróxidos dispersos. Este hecho se refleja en color de la muestra, ya que son estas las que presentan tonalidades más oscuras, con tonos marrones princi- 
Gisbert, J. et al., 2017. Caracterización y principales patologías de las “Piedra Dorada”... Boletín Geológico y Minero, 128 (2): $379-393$

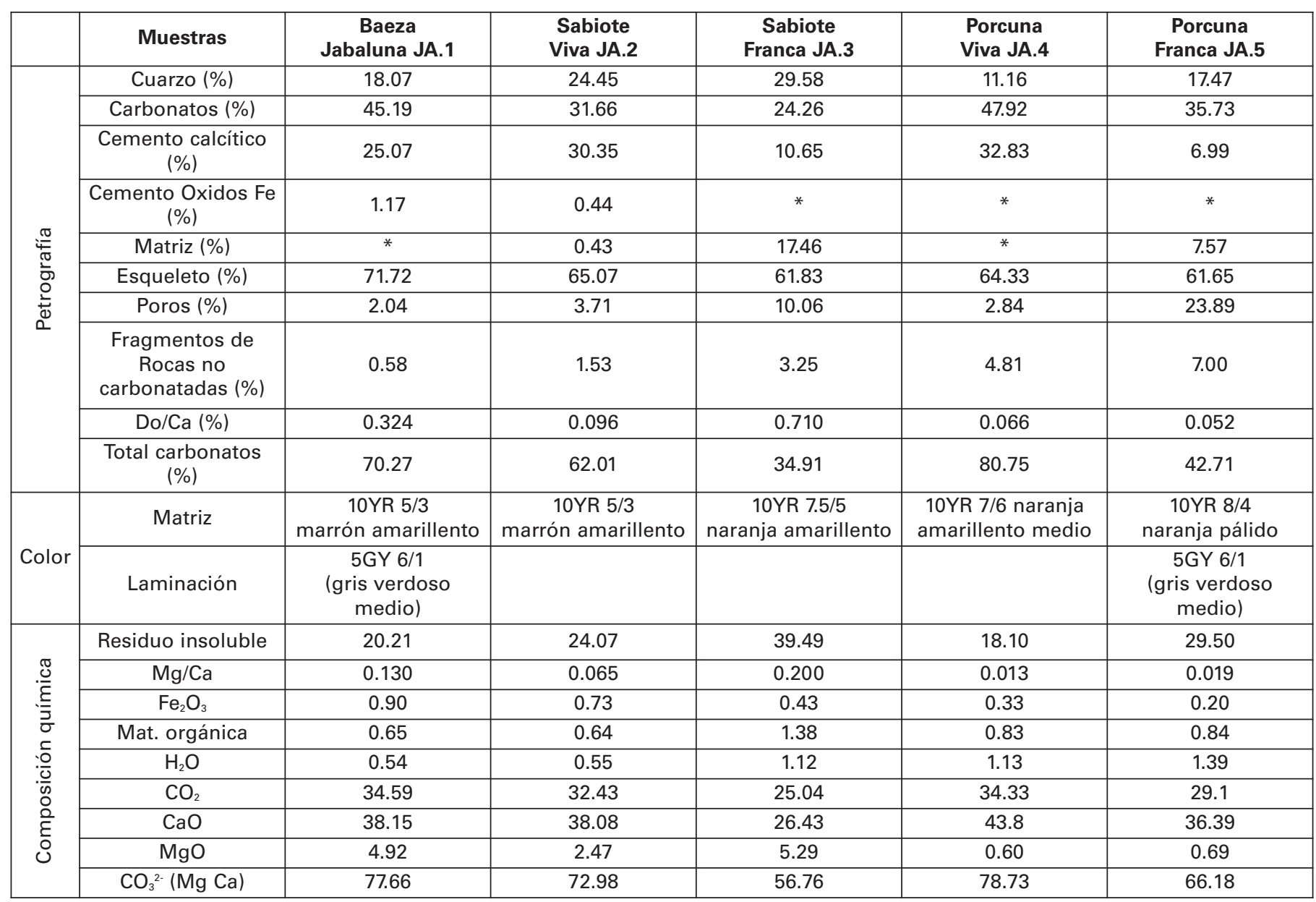

Tabla 1. Características petrográficas y análisis químicos de las muestras de cantera de "Piedra Dorada" (modificada de Gisbert et al., op. cit.). Matriz: Todos las partículas inferiores a $0.06 \mathrm{~mm}$.

Table 1. Petrographic features and chemical analysis of samples from the quarries were "Piedra Dorada" is extracted (modified from Gisbert et al., op. cit.). Matrix: All particles less than $0.06 \mathrm{~mm}$.

palmente. Además presentan mayor proporción de fragmentos dolomíticos que las muestras de Porcuna (JA.4). La variedad "Franca" de Sabiote (JA.3) presenta un porcentaje de fragmentos silíceos mayor que la de Porcuna (JA.5), que destaca por tener una laminación muy marcada frente a las otras rocas (Gisbert et al., op. cit.).

Con respecto a las variedades, la "Jabaluna" y "Viva" son poco porosas y están constituidas por microporos principalmente. Presentan un porcentaje elevado de cemento calcítico y un menor porcentaje relativo de residuo insoluble. Se pueden considerar el mismo tipo de roca, con excepción del color gris-azulado de "Jabaluna" (menos oxidada que "Viva"). La variedad "Franca" presenta una porosidad elevada y un doble sistema de porosidad que es su mayor debilidad, puesto que los macroporos facilitan la penetra- ción de agua y sales en los microporos, donde estos componentes actúan (Gisbert et al., op. cit.).

\section{Propiedades hídricas}

En lo referente al comportamiento frente al agua de las muestras de cantera, como se puede observar en la tabla 2, las variedades "Jabaluna" (procedente de Baeza) y "Viva" (de Sabiote y Porcuna) tienen una reducida porosidad accesible al agua con valores inferiores al $7 \%$; frente a los valores superiores al $22 \%$ que tiene la variedad "Franca" (de Sabiote y Porcuna).

La comparación de las gráficas que contienen la cinética de penetración del agua en el interior de las probetas permite observar claramente, el distinto 

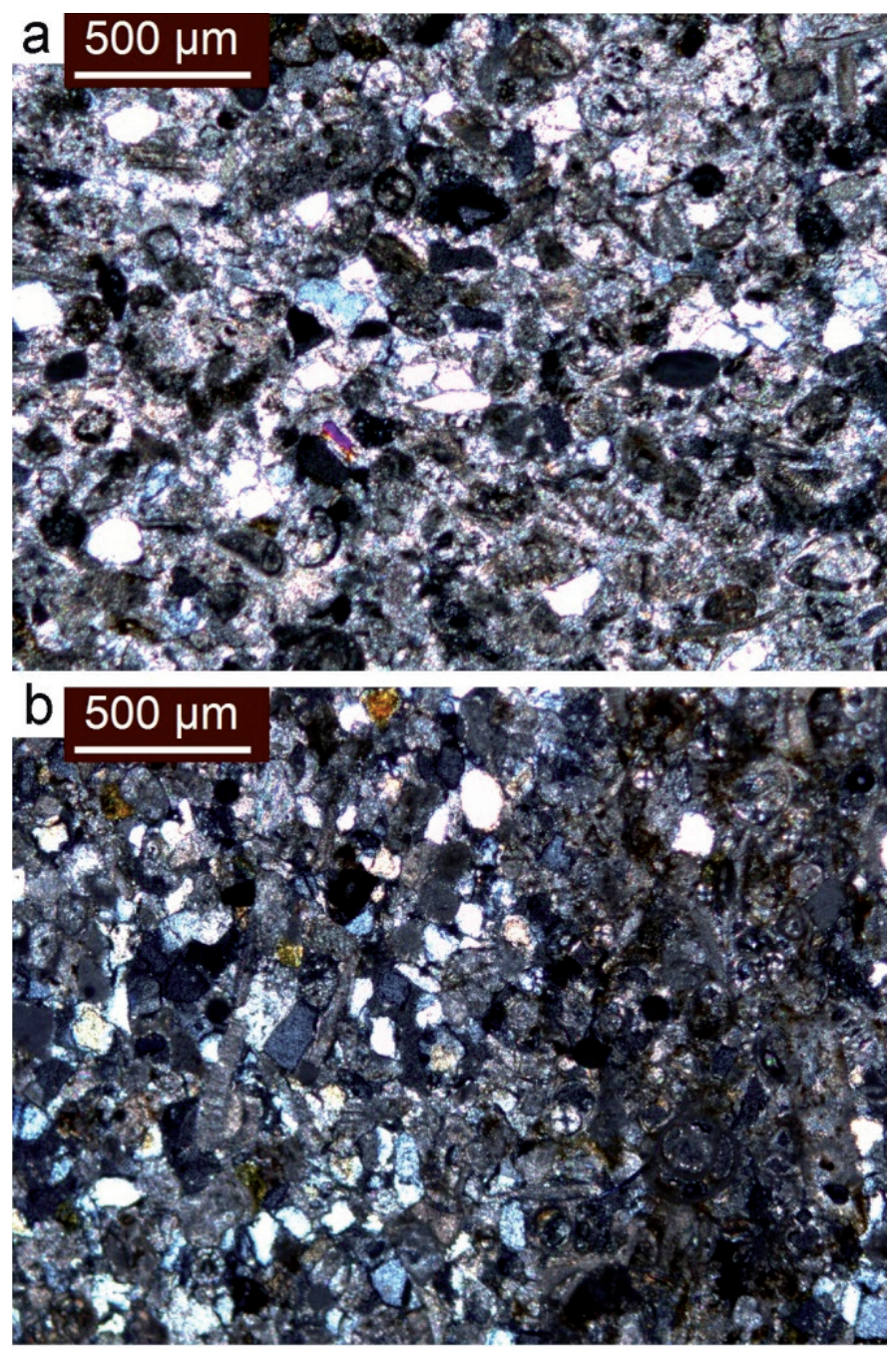

Figura 2. Microfotografías al MOP (con nícoles cruzados) de dos de las variedades estudiadas: a) Viva; b) Franca.

Figure 2. OPM photomicrographs (with crossed nicols) of two varieties studied: a) Viva; b) Franca.

comportamiento entre los dos grupos diferenciados con anterioridad (Fig. 3 a). Las variedades "Jabaluna" y "Viva" presentan una gráfica muy tendida y situada en valores inferiores al $3 \%$. Por un lado, la escasa pendiente de esta gráfica representa la baja velocidad de penetración del agua (variaciones de peso muy pequeñas frente al tiempo) y por otro, el bajo porcentaje absoluto apunta a la reducida absorción de agua. Por el contrario, las gráficas obtenidas para la variedad "Franca" destacan por los altos valores absolutos alcanzados y por la elevada velocidad con la que ha penetrado el agua en su interior. Además se pueden distinguir dos tramos distintos; el primero corresponde a una tendencia lineal de pendiente muy elevada y el segundo se caracteriza por una recta (aproximada) de pendiente baja. Por lo tanto, se puede aseverar que existe una relación directa entre variación de peso y porcentaje de poros conectados en el interior de la roca. A mayor variación absoluta, mayor porcentaje de poros conectados. Respecto a la velocidad de penetración del agua, la variedad "Franca" tiene un tramo inicial de gran pendiente que representa un elevado porcentaje de poros $y$, probablemente, de gran tamaño, ya que es necesaria una gran interconexión para que el agua circule tan rápido en el interior de la roca. Una vez saturados de agua estos poros empiezan a saturarse los de menor tamaño con lo cual el aumento de peso se ralentiza y la pendiente de la gráfica disminuye drásticamente. Respecto a las otras variedades, se observa como su gráfica presenta una pendiente casi homogénea. De esta morfología cabe inducir un sistema poroso formado por huecos de tamaño pequeño y homogéneo, y con escasa interconexión entre sí.

El ensayo de desorción nos ofrece una información que redunda en estos aspectos (Fig. 3 b). En las variedades "Jabaluna" y "Viva" tenemos una pérdida del agua retenida en sus poros muy lenta, frente a la observada en la variedad "Franca". Este dato reafirma la observación de que el sistema poroso es muy diferente, tanto en cantidad como en tortuosidad, siendo el porcentaje y el grado de interconexión mucho más elevados en la variedad "Franca". También se puede apreciar que el agua retenida al finalizar el ensayo es muy poca, aspecto que resulta positivo para todos los tipos litológicos.

Estos datos concuerdan con la bibliografía previa existente (Sebastián-Pardo et al., 1995). Estos autores realizaron un estudio del sistema poroso mediante ensayos petrofísicos y porosimetría de inyección de mercurio exclusivamente con las variedades "Viva" y "Franca". Los resultados cuantitativos coinciden con los obtenidos aquí, estableciendo además una serie de rangos de tamaño medio de poros para cada una de las variedades. Para la variedad "Viva", este tamaño se situaría entre 170 y $197 \mathrm{~nm}$ y en la variedad "Franca" en el rango que oscila entre 10470 y $11930 \mathrm{~nm}$.

\section{Comportamiento frente al deterioro}

Los resultados de este ensayo indican una relación directa entre el porcentaje de porosidad abierta y el deterioro sufrido por las probetas (tabla 3). Por lo tanto y a grandes rasgos, las variedades "Jabaluna" y "Viva" (Fig. 4 a, b y c) presentan un deterioro mucho menos acusado que la variedad "Franca" (Fig. $4 \mathrm{~d}$ y e). 
Gisbert, J. et al., 2017. Caracterización y principales patologías de las “Piedra Dorada”... Boletín Geológico y Minero, 128 (2): $379-393$

\begin{tabular}{|l|c|c|c|c|c|}
\hline Muestras & $\begin{array}{c}\text { Baeza } \\
\text { Jabaluna JA.1 }\end{array}$ & $\begin{array}{c}\text { Sabiote } \\
\text { Viva JA.2 }\end{array}$ & $\begin{array}{c}\text { Sabiote } \\
\text { Franca JA.3 }\end{array}$ & $\begin{array}{c}\text { Porcuna } \\
\text { Viva JA.4 }\end{array}$ & $\begin{array}{c}\text { Porcuna } \\
\text { Franca JA.5 }\end{array}$ \\
\hline Densidad aparente $\left(\mathrm{kg} / \mathrm{m}^{3}\right)$ & 2650 & 2526 & 1854 & 2461 & 1845 \\
\hline Densidad real $\left(\mathrm{kg} / \mathrm{m}^{3}\right)$ & 2702 & 2624 & 2508 & 2636 & 2380 \\
\hline Porosidad abierta (\%) & 1.92 & 3.75 & 26.05 & 6.65 & 22.47 \\
\hline Coeficiente absorción (\%) & 0.72 & 1.48 & 14.02 & 2.70 & 12.15 \\
\hline
\end{tabular}

Tabla 2. Resultados de los ensayos hídricos realizados a las muestras de cantera.

Table 2. Hydric test values of the samples from the quarries.
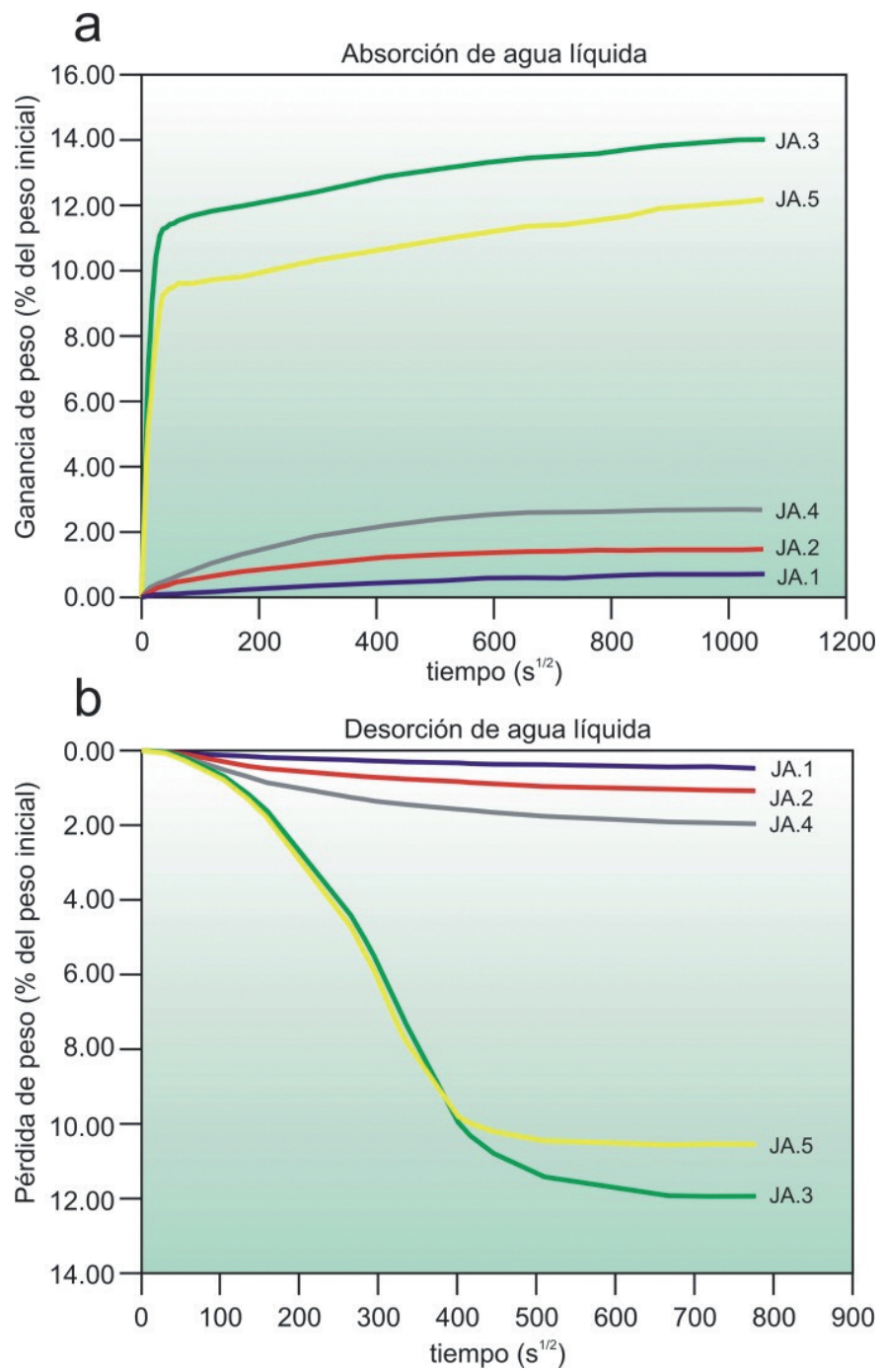

Figura 3. Cinética de penetración del agua en el interior de las probetas ensayadas. Absorción (a) y desorción (b).

Figure 3. Kinetics of water penetration into the tested samples. Absorption (a) and desorption (b).

Las variedades "Jabaluna" y "Viva" procedente de Sabiote se han deteriorado menos que la variedad "Viva" procedente de Porcuna, ya que esta última variedad presenta una anisotropía planar en forma de laminación paralela de escala milimétrica. En estos planos de anisotropía, más porosos que el resto de la roca, se han ido acumulando las sales solubles y ello conlleva un mayor deterioro. Este hecho ha generado la fractura de la roca en lajas. Esta fractura se ha generado en el proceso de disolución de las sales incluidas en el interior de la roca. Por otro lado, la mayor porosidad abierta así como la menor proporción de cemento de las variedades de "Franca" respecto a las "Vivas" o "Jabaluna" y su mayor proporción de matriz va a incrementar su alterabilidad, ya que la matriz no va a ejercer ningún papel aglomerante.

También se aprecia una diferencia en el comportamiento de la piedra "Franca" procedente de Sabiote frente a la proveniente de Porcuna. La primera presenta un deterioro más elevado que la segunda. Sin embargo, este deterioro se distribuye homogéneamente en las probetas de piedra "Franca" de Sabiote, mientras que en el otro tipo, la distribución no es uniforme. En este caso, la alteración viene determinada por la presencia de una laminación composicional y textural que induce al deterioro a distribuirse en forma de bandas, progresando más en las zonas menos resistentes y menos en las más duras.

Una vez definida las características y durabilidad de las distintas variedades de arenisca, se puede establecer el uso más adecuado de cada una de ellas. Esto permite un mayor aprovechamiento de la piedra y un ahorro económico en obras, al utilizarse piedra más adecuada en cada situación. El material utilizado en la construcción de los monumentos de Úbeda y Baeza ha sido principalmente, sillares de arenisca de las variedades "Viva" y "Franca", en la mayoría de edificios y en cualquier zona arquitectónica, mientras que la variedad "Jabaluna", ha sido utilizada principalmente para la construcción de zócalos, aunque también se pueden encontrar sillares aislados a diferentes alturas.

Gracias al estudio petrográfico y petrofísico se puede establecer una gradación en calidad del material para la construcción, que sería de mejor a peor 


\begin{tabular}{|l|c|c|c|c|c|}
\hline Muestras & $\begin{array}{c}\text { Baeza } \\
\text { Jabaluna JA.1 }\end{array}$ & $\begin{array}{c}\text { Sabiote } \\
\text { Viva JA.2 }\end{array}$ & $\begin{array}{c}\text { Sabiote } \\
\text { Franca JA.3 }\end{array}$ & $\begin{array}{c}\text { Porcuna } \\
\text { Viva JA.4 }\end{array}$ & $\begin{array}{c}\text { Porcuna } \\
\text { Franca JA.5 }\end{array}$ \\
\hline$\%$ pérdida de peso & -0.03 & 0.07 & -41.22 & -5.03 & -19.64 \\
\hline
\end{tabular}

Tabla 3. \% de Pérdida de peso obtenida tras el ensayo de cristalización de sales.

Table 3. \% of loss of weight after the salt crystallization test.

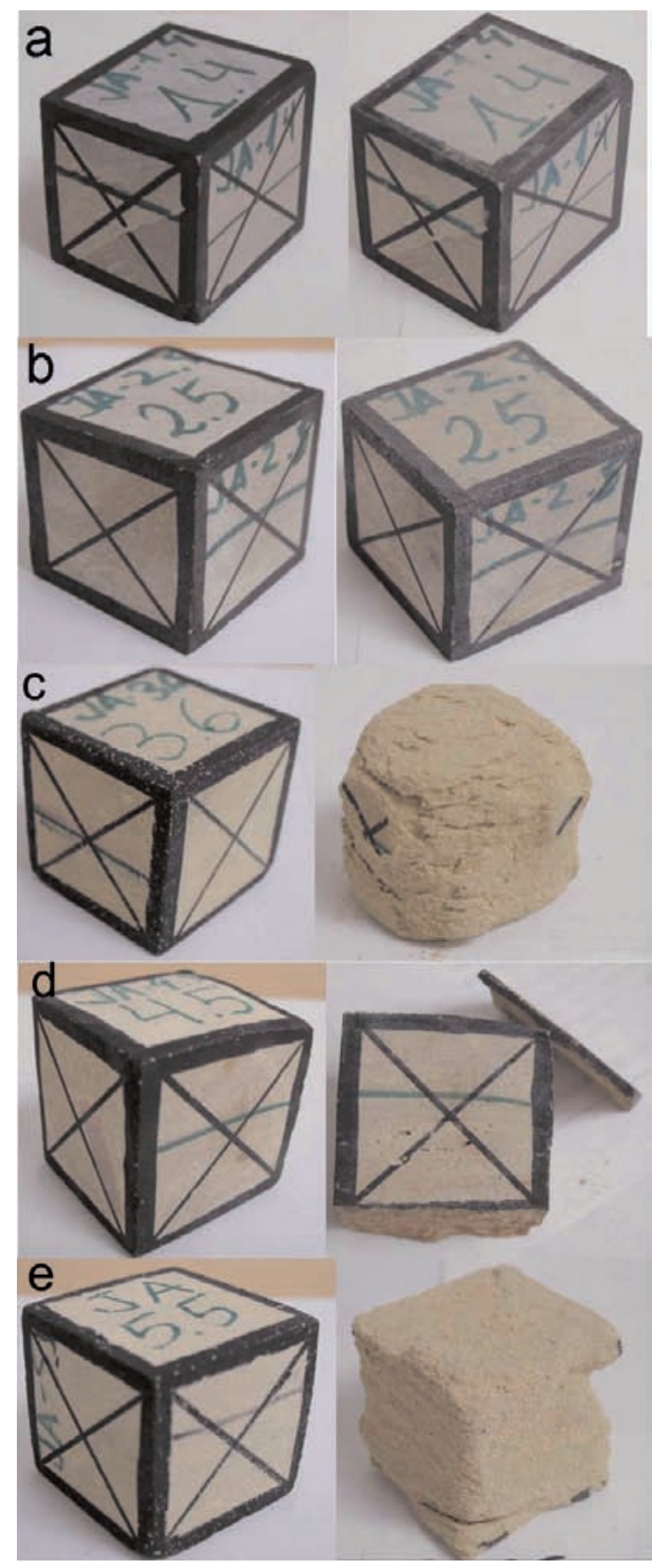

Figura 4. Aspecto inicial y final de las probetas ensayadas frente al ensayo de deterioro: a) Jabaluna Baeza JA.1; b) Viva Sabiote JA.2; c) Franca Sabiote JA.3; d) Viva Porcuna JA.4; e) Franca Porcuna JA.5.

Figure 4. Initial and final aspect of tested samples against the deterioration test: a) Jabaluna JA.1 Baeza; b) Viva Sabiote; JA.2 c) Franca Sabiote; JA.3 d) Viva Porcuna; JA.4 e) Franca Porcuna JA.5. calidad por este orden la "Jabaluna", "Viva" de Sabiote, "Viva" de Porcuna, "Franca" de Porcuna y "Franca" de Sabiote.

\section{Principales patologías}

En cuanto a los deterioros diagnosticados en los edificios estudiados, cabe destacar: colonización biológica (líquenes, algas, musgos y plantas superiores), humedades y depósitos superficiales (sales y costras negras).

En la Fig. 5 se muestran algunos ejemplos de los deterioros de los monumentos estudiados.

A continuación se detallan las características de las costras negras y eflorescencias estudiadas:

a) Costras negras

En la muestra U.1, procedente de la Iglesia de San Salvador (Úbeda) se ha estudiado una costra negra desarrollada en el lado izquierdo de la portada norte. El análisis ha mostrado un alto grado de colonización tanto en densidad como en profundidad. Se han encontrado grandes colonias de líquenes en superficie $(0,5 \mathrm{~mm}$ de espesor) cuyos talos penetran a través de toda la muestra observada ( $5 \mathrm{~mm}$ ) (Fig. 6 a). Las observaciones y el hecho de que esta muestra procediera de una escama prácticamente desprendida de la pared indican una elevada intensidad del deterioro físico-químico inducido por estos organismos.

En la muestra U.2 procedente de la portada norte de la misma iglesia se ha estudiado el desarrollo de una costra sobre dos soportes, roca arenisca del monumento y mortero de cal en la junta de unión de los sillares. Las observaciones indican una costra negra mixta de contaminación y biogénica de muy poco desarrollo. Se han encontrado elementos de ambos, cristales de yeso, partículas esféricas de contaminación y restos biológicos (Fig. 6 b y c). Es posible que esta costra de muy escaso desarrollo solo se encuentre en el interior de la portada al ser una zona más abrigada que el resto del lado Norte, y favorezca el depósito de partí- 

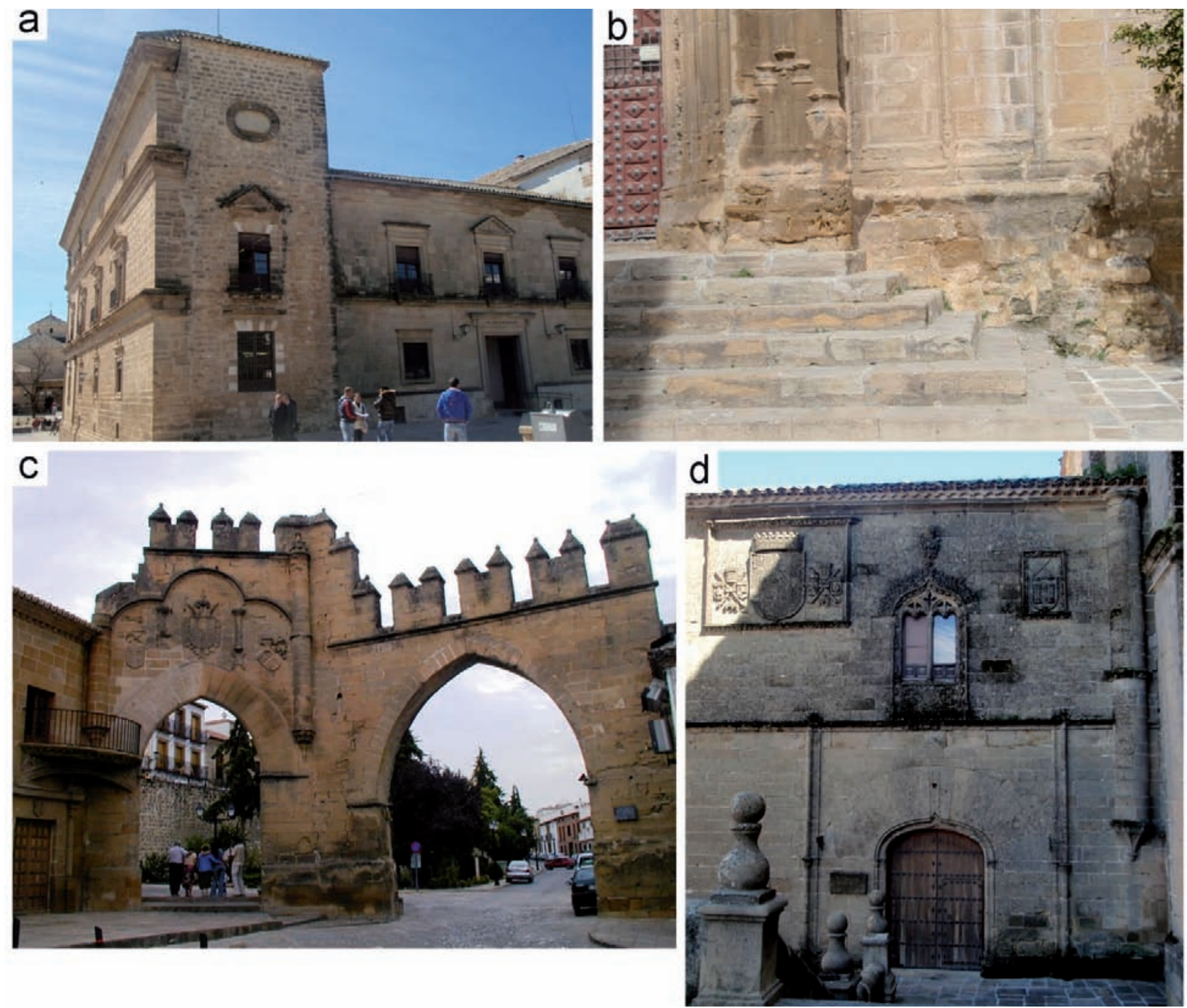

Figura 5. Algunos ejemplos de deterioros observados: a) Colonización biológica en la cornisa y en la zona de la bajante, en el Ayuntamiento de Úbeda; b) Deterioro del exterior de la iglesia de San Pablo (Úbeda) en el que se observa un fuerte proceso de arenización y/o erosión alveolar y eflorescencias; c) Puerta de Jaén y Arco de Villalar (Baeza), donde destaca la presencia de costras negras; d) Catedral de Baeza, donde se observa gran cantidad de colonización biológica, eflorescencias y costras negras, entre otros deterioros.

Figure 5. Examples of deterioration observed: a) biological colonization on the ledge and in the downpipe, in the city of Úbeda; b) deterioration of the exterior of the church of San Pablo (Úbeda) showing a strong process of grain disaggregation and/or alveolar erosion and efflorescence; c) Gate of Jaén and Arch of Villalar (Baeza), with the presence of black crusts which highlights the decay of the Gate of Jaen and Arch of Villalar (Baeza); d) Cathedral of Baeza, where many biological colonizations, black crusts and efflorescence are observed, among other damage.

culas al no producirse un lavado por parte de la Iluvia.

La muestra B.2, se tomó del zócalo de la esquina noroeste del Arco de Villalar (Baeza), es de gran interés debido al tráfico rodado que atraviesa el arco. Se ha encontrado una capa de unas $100 \mu \mathrm{m}$ de espesor, con una composición similar a la encontrada en la muestra U.2, pero con un mayor desarrollo. Se observa una zona con cristales de yeso en forma de rosa del desierto (Fig. $6 \mathrm{~d}$ ) junto a restos de la roca disgre- gada. Esta zona porosa facilita la retención de partículas de contaminación y la colonización de organismos.

b) Eflorescencias salinas

Con respecto a las eflorescencias salinas, tras su análisis mediante difracción de rayos $X$ se observa que las fases principales encontradas son principalmente sulfatos de magnesio, níter (nitrato potásico, $\mathrm{KNO}_{3}$ ), cuarzo y calcita que aparecen en casi todas las muestras. Otras 

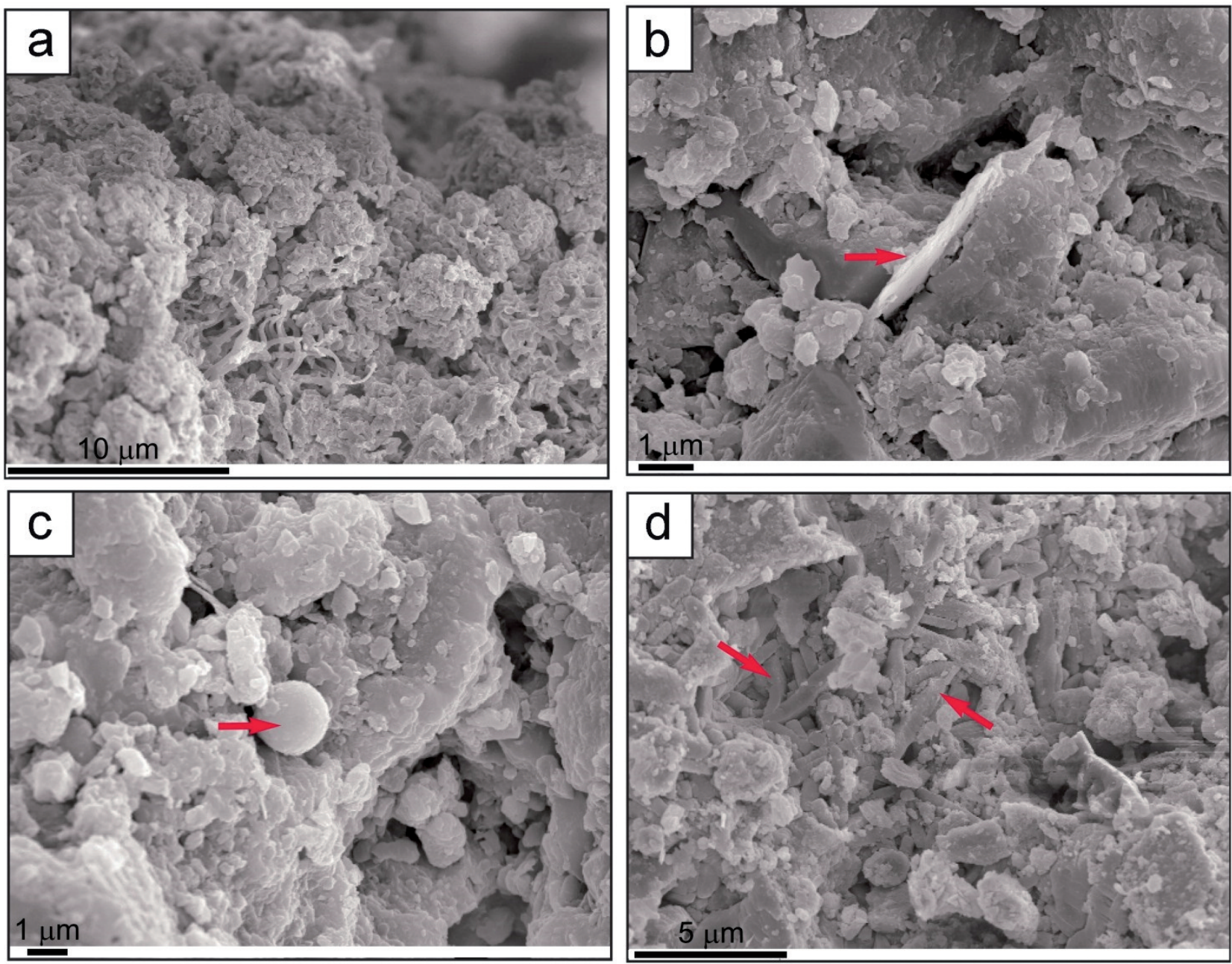

Figura 6. Aspecto de algunas de las muestra de costras negras estudiadas: a) filamentos y formas globulares de origen orgánico adheridos al sustrato de la muestra U.1; b) cristales de yeso autígenos formados por procesos de disolución/precipitación en el interior de una costra negra de la muestra U.2: c) partículas esféricas características procedentes de la contaminación por combustión de carbón de la muestra U.2; d) Vista de la superficie externa de la muestra B.1, donde se aprecian cristales de yeso en forma de rosa del desierto. Esta zona porosa facilita la retención de partículas contaminantes y de organismos. Los componentes de fondo son calcíticos.

Figure 6. Aspect of some black crusts of studied samples: a) filaments and globular organic origin attached to the substrate of the sample $U .1 ; b)$ authigenous gypsum crystals formed by dissolution / precipitation inside a black crust of the sample U.2; c) characteristic spherical particles of pollution from coal combustion of the sample U.2; d) view of outer surface of the sample B.1, where gypsum crystals are seen as desert roses. This porous zone facilitates retention of particulate pollutants and organisms. The background components are of calcitic composition.

mineralogías presentes son nitrato potásico $\left(\mathrm{NaNO}_{3}\right)$ y yeso $\left(\mathrm{CaSO}_{4}\right) \cdot 2 \mathrm{H}_{2} \mathrm{O}$, halladas en una sola muestra cada una. En la tabla 4 se muestran las principales fases encontradas en cada muestra.

Los sulfatos de Mg están presentes en cinco de las seis muestras analizadas y aparecen según distintas estructuras, principalmente hexahedrita $\left(\mathrm{MgSO}_{4} \cdot 7 \mathrm{H}_{2} \mathrm{O}\right)$ o epsomita $\left(\mathrm{MgSO}_{4} \cdot 6 \mathrm{H}_{2} \mathrm{O}\right)$. Esta abundancia y su mineralogía indican la existencia desde el subsuelo de una fuente rica en sulfato que disuelve parcialmente la roca (rica en dolomita, carbonato de $\mathrm{Mg}$ ) o bien que provengan del lavado de las abundantes reposiciones con cemento Portland, dando como resultado que este tipo de sales sea mayoritario. Probablemente, este sea el mismo origen para el yeso presente en la muestra B.6, ya que el sustrato también es muy rico en calcio. Este tipo de sales tienen un efecto muy perjudicial en la roca ya que son muy higroscópicas, es decir tienen una elevada capacidad para captar humedad en forma de vapor de agua de la atmósfera. Esta característica genera un mayor 
Gisbert, J. et al., 2017. Caracterización y principales patologías de las “Piedra Dorada”... Boletín Geológico y Minero, 128 (2): $379-393$

\begin{tabular}{|c|c|c|c|c|c|c|}
\hline Muestra & Sulfatos de Mg & Níter & Nitrato sódico & Yeso & Cuarzo & Calcita \\
\hline Ayto. de Úbeda (U.23) & $\mathrm{X}$ & $\mathrm{X}$ & & & $\mathrm{x}$ & $\mathrm{X}$ \\
\hline Convento de S. Francisco (B.37) & $x$ & $x$ & & & $x$ & $\mathrm{x}$ \\
\hline Palacio de los Condes de Guadiana (U.5) & $\mathrm{x}$ & & & & $x$ & $\mathrm{x}$ \\
\hline Iglesia de San Pablo (U.7) & & $x$ & $\mathrm{x}$ & & $\mathrm{x}$ & \\
\hline Catedral de Baeza (B.6) & $x$ & & & $\mathrm{X}$ & $\mathrm{x}$ & $\mathrm{x}$ \\
\hline
\end{tabular}

Tabla 4. Principales fases encontradas en las muestras de eflorescencias salinas en los monumentos de Úbeda y Baeza.

Table 4. Main phases found in the samples of salt efflorescence on the monuments of Úbeda and Baeza.

aporte de humedad al muro, lo cual provoca a su vez un mayor número de ciclos de cristalización de sales que pueden deteriorar la piedra y producir las antiestéticas manchas oscuras presentes en la mayoría de las fachadas.

Los nitratos están presentes en cuatro de las seis muestras. En su estructura se concentran los cationes monovalentes (potasio y sodio) movilizados por las humedades, mientras que los divalentes (calcio y magnesio) aparecen ligados a los sulfatos. En principio, el origen sería similar al del caso anterior: un ascenso capilar procedente del subsuelo rico en nitratos y del lavado de reposiciones con cemento Portland. En este caso, la roca no parece ser una fuente de cationes por lo que tendrían que estar disueltos en origen en las aguas que inducen las humedades. En cualquier caso, podría relacionarse la concentración de nitratos en las aguas del subsuelo debido a la intensa actividad agrícola de la zona. Por último, merece especial atención la muestra U.7 en la cual existen únicamente nitratos. Este hecho puede indicar una fuente de humedad distinta al resto. La elevada concentración de nitratos se debería a un aporte orgánico como aguas procedentes de cubiertas que hubieran lixiviado guanos de aves.

El cuarzo al ser elemento muy poco móvil es un resto de la propia roca alterada. Además, suele tener una intensidad de reflexión muy elevada, por lo que aunque este en baja proporción aparece con mucha claridad.

La calcita es un mineral más soluble que el cuarzo y, por tanto, más móvil. Este hecho provoca que la calcita que aparece en los difractogramas no se sepa si es un elemento de la roca alterada o si ha precipitado al estar diluida en las humedades que pudieran haber afectado al muro.

El posible origen de estas sales está en el ascenso capilar de agua del suelo a través del sustrato, que aporta nitratos y sulfatos, así como en el lavado de zonas ricas en sulfatos y nitratos como zonas repuestas con cemento Portland. La abundancia de magnesio en la roca genera sales de magnesio que poseen una elevada higroscopicidad. Esto se traduce en la presencia de manchas de humedad que solo pueden eliminarse mediante la retirada de estas sales del interior de la roca.

Los deterioros en las fábricas a una altura de entre 2 y 3 metros se deben principalmente a la colocación de piedra "Viva" o "Jabaluna" en las primeras hileras de sillares así como en la cimentación, lo que conduce a una fácil e importante ascensión capilar de las sales solubles, debido, principalmente a su microporosidad, hasta esas zonas de 2 o 3 metros en los que aparecen piedras más porosas, frecuentemente la "Viva", con menor proporción de microporosidad (Sebastián-Pardo et al., op cit.). Los resultados observados son similares a los obtenidos por otros autores en iglesia de San Pablo de Úbeda. Campos y de la Torre (2007) pusieron de manifiesto que las costras negras estudiadas, que se encuentran en zonas protegidas, en las que la lluvia no incide directamente (elementos ornamentales, cornisas, molduras, etc.) están formadas principalmente por un entramado de yeso acicular. En las eflorescencias aparecían, al igual que en los casos aquí estudiados, sulfatos y nitratos (Campos y de la Torre, 2006), procedentes de la disolución de excrementos de pájaro así como otras formas de contaminación biológica en los sillares infe- 
riores. Estos autores achacan estos fenómenos a que los sillares de la iglesia de San Pablo presentan una elevada capacidad de ascenso de agua por capilaridad que favorece la circulación de iones solubles hasta niveles elevados de la estructura. La presencia de cloruros es especialmente significativa en la fachada que cuenta con una pila adosada de agua potable.

\section{Conclusiones}

En cuanto a la caracterización de la roca podemos concluir que las variedades "Jabaluna" y "Viva" son muy poco porosas y con un sistema de huecos formado por microporos y un elevado porcentaje de cemento calcítico. Se pueden considerar el mismo tipo de roca con el matiz de que la variedad "Jabaluna" está menos oxidada presentando un color gris-azulado. La variedad "Franca" es muy porosa, constituida por macroporos y microporos. Este doble sistema de poros la hace más débil, al penetrar sales en los microporos y agua en los macroporos, y por tanto es susceptible de un mayor deterioro. En ocasiones hay laminación en la roca que se corresponden con planos de debilidad (ejemplos más comunes en Porcuna).

Por lo tanto se puede afirmar que las variedades "Viva" y "Jabaluna" más cementadas y duras, son mejores para el uso con propósitos estructurales (sillares, columnas, capiteles, etc.) y la variedad "Franca", menos cementada es más apta para elementos constructivos no estructurales, es decir, para fines ornamentales (fachadas, estatuas, etc.).

Los deterioros diagnosticados en los edificios estudiados son: colonización biológica (líquenes, algas, musgos y plantas superiores), humedades y depósitos superficiales (sales y costras negras).

A partir del estudio de las costras superficiales se puede deducir que la contaminación es poco intensa y da problemas en puntos muy concretos del centro de ambas ciudades, como se ha puesto de manifiesto la casi nula aparición de partículas contaminantes en las poblaciones muestreadas. Las humedades en zócalos perimetrales son frecuentes y es el principal problema de deterioro. Esta procede tanto de ascensos capilares del sustrato como de salpiqueos de cubiertas.

Otra de las causas más importantes de deterioro son las humedades originadas por el movimiento del agua que contiene iones disueltos y que precipitan en el interior del material pétreo a unos pocos micrómetros de la superficie. La composición de las sales corresponde mayoritariamente a sulfatos y a nitratos.
Los primeros son de magnesio o de calcio y los segundos de potasio o de sodio. El posible origen de estas sales está en el ascenso capilar de agua del suelo a través del sustrato formado por rocas con un mayor desarrollo de microporosidad, que aporta nitratos y sulfatos, así como en el lavado de zonas ricas en sulfatos y nitratos como zonas repuestas con cemento Portland o de cubiertas con restos de guano.

Los materiales estudiados presentan un deterioro natural intrínseco a las propias características del material con el que se construyó, debido al inevitable paso de los años. La desaparición de las canteras históricas de las que se extrajeron los materiales puede suponer un problema para el futuro dado el escaso conocimiento actual sobre los materiales que presentan características más análogas a los empleados. Por tanto, una de las prioridades de los diferentes organismos encargados de la protección del patrimonio debería ir centrada en la recuperación de las canteras históricas o en la búsqueda de un material apto para la restauración y reparación de los principales monumentos, lo más similar posible al original. Asimismo, centrar futuras investigaciones en conocer qué productos de tratamiento son los más compatibles con este material de construcción y en qué medida mejora su durabilidad, se estima como una herramienta de protección fundamental. Todas estas actuaciones, generarían posibilidades de emprender nuevas explotaciones por la zona o ampliar las ya existentes, contribuyendo a la economía de la zona.

\section{Agradecimientos}

Este trabajo ha sido realizado en el marco del convenio IGME-Diputación de Jaén: "Estudio de patologías de la piedra y diagnóstico del estado del patrimonio histórico-monumental de la comarca de La Loma". Además ha sido en parte financiado a través de CONSTRUROCK (Red de la Piedra Natural y su relación con el Patrimonio Histórico-Monumental y la obra nueva, en el ámbito del Estado español). Los autores también quieren agradecer a dos revisores anónimos sus correcciones y sugerencias para ayudar a mejorar este artículo.

\section{Referencias}

Alcalde, M., Terreros, G.G. and Villegas, R. 1998. Morfología macroscópica de alteración de la piedra de la Catedral de Baeza, Jaén (España). Materiales de Construcción, 48 (252), 27-44.

Alfaro, P., Molina, J.M., Moretti, M. and Soria, J.M. 1996. Estructuras sedimentarias de deformación originadas 
por licuefacción en depósitos de tormentas (Mioceno de la Cuenca del Guadalquivir, provincia de Jaén). Geogaceta, 20 (2), 311-314.

Beard, D.C. y Weyl, P.K. 1977. Influence of texture on porosity and permeability of unconsolidated sand. En: Syed A.A. y Friedman, G.M. "(ed)", Diagenesis of sandstones. AAPG Reprint Series, Tulsa, Oklahoma, 186-206.

Campos, M.J. y De la Torre, M.J. 2006. Composición y origen de las sales en la iglesia de San Isidoro (Úbeda). Macla, 6, 119-122.

Campos, M.J. y De la Torre, M.J. 2007. Costras negras y procesos de alteración superficial en la iglesia de San Pablo (Úbeda). Macla, 7, 19.

Charola, A.E. 2004. Stone deterioration in historic buildings and monuments, Proceedings of the $10^{\text {th }}$ International Congress on Deterioration and Conservation of Stone. ICOMOS, 3-14. Kwiatkowski, D. y Löfvendahl, R.(eds) Stockholm, 3-14.

Cultrone, G., Luque, A. y Sebastián-Pardo, E. 2012. Petrophysical and durability test on sedimentary stones to evaluate their quality as building materials. Quarterly Journal of Engineering Geology and Hydrogeology, 45, 415-422.

García-Torralbo, M.C. 1998. Baeza conventual: el espacio conventual en el contexto urbano de Baeza en los siglos XVI y XVII. Universidad de Jaén. 495 pp.

Geological Society of America, 1991. Rock color chart with genuine Munsell color chips. G. S. A. 4 p + 6 láminas.

Gisbert, J., Franco B., Mateos I., Colucci, F., Baltuille, J.M. Buj, O. 2007. Caracterización petrográfica de los materiales empleados en los monumentos de Úbeda y Baeza. Macla, 8, 1.

Luque, A., Cultrone, G., Sebastián-Pardo, E. y Cazalla, O. 2008. Effectiveness of stone treatments in enhancing the durability of bioclastic calcarenite in (Granada, Spain). Materiales de Construcción, 58 (292), 115-128.

Molina, E., Cultrone, G., Sebastián-Pardo, E. y Alonso, F.J. 2013. Evaluation of stone durability using a combination of ultrasound, mechanical and accelerated aging tests. Journal of Geophysics and Engineering 10, DOI: 10.1088/1742-2132/10/3/035003.

Molina, E., Cultrone, G., Sebastián-Pardo, E., Alonso, F. J., Carrizo, L., Gisbert, J. and Buj, O. 2011. The pore system of sedimentary rocks as a key factor in the durability of building materials. Engineering Geology, 118, 110-121.

Nimis, P. L., Pinna, D. and Salvadori, O. 1992. Licheni e conservazione dei monumenti. Editrice CLUEB, Bologna (Italia), $170 \mathrm{pp}$.

RILEM 1980. Essais recommandés pour mesurer l'altération des pierres et évaluer l'eficacité des méthodes de traitements. RILEM Bulletin 13 (75), 175-252.
Rodríguez-Navarro, C. 1994. Causas y mecanismos de alteración de los materiales de calcáreos de las Catedrales de Granada y Jaén. Tesis doctoral. Universidad de Granada, 412 pp.

Rodríguez-Navarro, C. y Doehne, E. 1999. Salt weathering: influence of evaporation rate, supersaturation and crystallization pattern. Earth Surface Processes and Landforms, 24 (3), 191-209.

Roldán, F.J. 1995. Evolución neógena de la cuenca del Guadalquivir, Tesis doctoral. Universidad de Granada, Granada, 259 pp. Inédita.

Roldán, F.J., 2011. La Andalucía Alpina. In: García-Cortés, A. “(ed)", Cartografía de recursos minerales de Andalucía. IGME-Consejería de Economía, Innovación y Ciencia de la Junta de Andalucía, Madrid, 32-60.

Ruiz-Agudo, E., Lubelli, B. Sawdy, A. van Hees, R. Price, C. y Rodriguez-Navarro, C. 2011. An integrated methodology for salt damage assessment and remediation: the case of San Jerónimo Monastery (Granada, Spain). Environmental Earth Sciences, 63, 1475-1486.

Saiz-Jiménez, C. y Ariño, X., 1995. Colonización biológica y deterioro de morteros por organismos fotótrofos. Materiales de Construcción, 45, 5-16.

Sebastián-Pardo, E. M., Cultrone, G., Garibaldi, V., Rodríguez-Navarro, C., De la Torre, M. J. and Valverde, I. 2008. Swelling damage in clay-rich sandstones used in the church of San Mateo in Tarifa (Spain). Journal of Cultural Heritage, 9, 66-76.

Sebastián-Pardo, E.M., Martín-Clabo, J. y Zezza, U., 1995. The "Piedra Dorada" Calcarenite in the cultural built heritage of Baeza (Andalusian Province of Jaén, Spain). Atti Ticinesi di Scienze della Terra, 38, 205-213.

UNE-EN-1936. 2007. Métodos de ensayo para piedra natural. Determinación de la densidad real y aparente y de la porosidad abierta y total. A.E.N.O.R. Madrid.

UNE-EN-13755. 2008. Métodos de ensayo para piedra natural. Determinación de la absorción de agua a presión atmosférica. Edited by A.E.N.O.R. Madrid.

Urosevic, M., Sebastián-Pardo, E.M., Ruiz-Agudo, E. y Cardell, C., 2011. Evaluación de las propiedades físicas de dos rocas carbonáticas usadas como material de construcción actual e histórico en Andalucía Oriental, España. Materiales de Construcción, 61 (301), 93-114.

Urosevic, M., Yebra-Rodríguez, A., Sebastián-Pardo, E. y Cardell, C., 2012. Black soiling of an architectural limestone during two-year term exposure to urban air in the city of Granada (S Spain). Science of Total Environment, $414,564-575$.

Watt, J., Tidblad, J., Kucera, V. y Hamilton, R., 2009. The effects of air pollution on Cultural Heritage, XII. Springer, New York, 299 pp.

Recibido: diciembre 2015

Revisado: marzo 2016

Aceptado: mayo 2016

Publicado: junio 2017 
